\title{
Acute liver failure
}

\author{
Authors: Mohammed A Arshad, ${ }^{A}$ Nicholas Murphy ${ }^{B}$ and Mansoor N Bangash ${ }^{C}$
}

Acute liver failure is a rare syndrome and is primarily caused by paracetamol toxicity in developed nations. Survival for patients with acute liver failure has steadily improved over the last few decades from approximately $20 \%$ to greater than $60 \%$. This marked improvement in survival has been due to a combination of improvements in medical practice and the use of emergency liver transplantation in selected patients. Early recognition and timely initial management in the nonspecialist centre can significantly improve outcomes. Patients should be simultaneously discussed with a transplant centre and referred to critical care. Close liaison with transplant centres to ensure timely transfer in deteriorating patients is important.

\section{Introduction}

Acute liver failure (ALF) is a rare syndrome with a UK incidence of 1-8 per million population. It comprises an acute reduction in metabolic capacity, characterised by a coagulopathy, jaundice and encephalopathy in a patient with no prior history of liver disease. ${ }^{1}$ In the UK, ALF is primarily caused by paracetamol overdose (POD). Less common aetiologies include viral hepatitis, drug-induced liver injury, pregnancy-induced liver failure and autoimmune hepatitis, but there is also a significant proportion that is due to indeterminate causes. ${ }^{2}$ Survival for patients with ALF has steadily improved over the last few decades. In the 1970s, roughly five in six ALF patients admitted to the intensive care unit (ICU) died, whereas more recently, the survival rates are $>60 \%{ }^{3}$ This marked improvement in survival has been due to a combination of changes in medical management and the use of emergency liver transplantation (ELT) in selected patients. Currently ELT is reserved for a subset of those unlikely to survive with medical care alone. The European Association for the Study of the Liver (EASL) has recently published comprehensive

Authors: ${ }^{A}$ anaesthetic and critical care consultant, Queen Elizabeth Hospital, Birmingham, UK and Birmingham Liver Failure Research Group, Birmingham, UK; ${ }^{B}$ anaesthetic and critical care consultant, Queen Elizabeth Hospital, Birmingham, UK, Birmingham Liver Failure Research Group, Birmingham, UK, and honorary reader, University of Birmingham, Birmingham, UK; ${ }^{C}$ anaesthetic and critical care consultant, Queen Elizabeth Hospital, Birmingham, UK, Birmingham Liver Failure Research Group, Birmingham, UK, and honorary senior clinical lecturer, University of Birmingham, Birmingham, UK evidence-based guidelines on the management of acute liver failure. ${ }^{4}$ In this article, we briefly review what the generalist needs to know about ALF.

\section{Classification}

The presentation of ALF varies according to aetiology, and aetiologies vary in their relative frequency across the globe. Consequently, several differing classification systems exist but essentially all differentiate between rapidly progressive ALF and other forms. This distinction holds prognostic significance. The UK tends to use the O'Grady classification. Patients present as either 'hyperacute', 'acute' or 'subacute' dependent upon the emergence of encephalopathy following the first detected symptoms, often jaundice (Fig 1). ${ }^{5}$ Hyperacute presentations frequently develop extrahepatic organ failure and carry a high risk of cerebral oedema and intracranial hypertension but, counterintuitively, carry the best prognosis with medical treatment alone. Acute and subacute presentations usually have a more indolent course over several weeks, extrahepatic organ failures develop

\section{Key points}

Acute liver failure is a rare syndrome comprising a coagulopathy of liver origin, jaundice and encephalopathy in someone without pre-existing liver disease.

Fluid resuscitation, administration of $\mathrm{N}$-acetyl cysteine regardless of aetiology and broad spectrum antibiotic and antifungal therapy should be initiated.

Early referral to critical care and discussion with a transplant centre is recommended for all patients.

King's College hospital criteria are currently used to identify those patients who would not survive without liver transplantation and are the basis of transplant criteria in the UK.

Survival from acute liver failure has increased due to improvements in medical management and appropriate use of emergency liver transplantation.

KEYWORDS: Acute liver failure, drug overdose, paracetamol, patient selection, liver transplantation

DOI: 10.7861/clinmed.2020-0612 


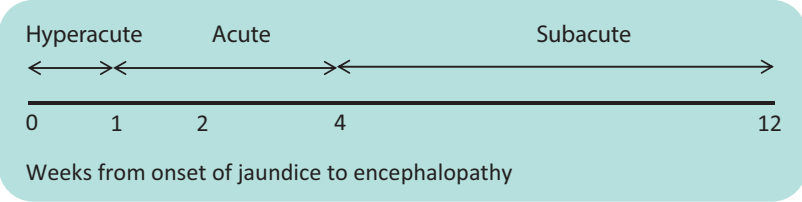

Fig 1. 0'Grady classification of acute liver failure. ${ }^{5}$

later and they have a lower risk of cerebral oedema. However, these patients are less likely to recover with supportive care alone and outcomes are poorer without liver transplantation. Possible exceptions to this include viral hepatitis where antiviral treatments improve outcomes but transplantation is usually still required if encephalopathy develops. ${ }^{6}$

\section{Making the diagnosis}

Recognising and diagnosing ALF in a timely fashion requires a high index of suspicion considering its rarity. It should be looked out for in all patients without pre-existing liver disease who develop or present with a liver injury (international normalised ratio (INR) $>1.5$ and transaminases $>3$ times the upper limit of normal). Hepatic encephalopathy in this context defines ALF and can be very subtle in early stages. Its presence should therefore be actively sought. In case of diagnostic uncertainty, an ammonia measurement may alert the clinician to a hepatic cause of somnolescence or confusion when the cause is not obvious. Elevated lactate can also point toward the diagnosis and serial lactate measurements can map the disease trajectory and should be undertaken. Identifying the cause of ALF is also important as it carries prognostic significance and alters management. Therefore, once ALF is suspected, the patient's history should be revisited and a liver disease screen done to determine cause (see Table 1).

Table 1. Initial investigations to help establish the cause of acute liver failure

Toxicology for drugs including paracetamol/salicylate

Viral hepatitis screen:

> hepatitis A, B, C, E

$>$ herpes simplex virus

$>$ varicella-zoster virus

Cytomegalovirus

$>$ Epstein-Barr virus

$>$ parvovirus.

Autoimmune hepatitis screen:

$>$ antinuclear antibodies, anti-smooth muscle antibodies, anti-soluble liver antigen, globulin profile, anti-neutrophil cytoplasmic antibodies.

Other investigations dependent upon clinical situation:

$>$ pregnancy test

$>$ ammonia measurement

> caeruloplasmin/slit-lamp examination for Kayser-Fleischer rings if Wilson's disease suspected

> bone marrow / lymph node biopsy if lymphoma suspected.

Doppler ultrasound / computed tomography of the liver to assess patency of portal vein, hepatic artery and hepatic veins.
Ultrasound to assess the vascular supply and drainage of the liver and assess for pre-existing liver disease is mandatory. Computed tomography (CT) may occasionally be required in order to exclude chronic liver disease and to estimate liver volume in those with subacute presentations.

\section{Initial management}

Initial management includes stopping hepatotoxic drugs, defending circulating volume using crystalloids ( $0.9 \%$ saline or a balanced crystalloid), initiating broad spectrum antibiotics and antifungals (these patients are highly susceptible to infection), and starting N-acetyl cysteine (NAC). ${ }^{4,7}$ NAC is historically associated with POD treatment but is now recommended for all ALF aetiologies due to probable benefit. ${ }^{8}$ Although the recent SNAP study showed that for POD presenting to the emergency department, a new 12-hour 300 mg/kg NAC regimen was better tolerated and non-inferior to an older 21-hour regimen in terms of consequent hepatic injury and ALF, there is still a lack of clarity regarding the optimal duration of NAC infusion once ALF has developed. ${ }^{9}$ Furthermore, caution is required in extrapolating the findings of this trial to delayed or staggered overdoses where outcomes tend to be worse.

Hepatic synthetic function is best monitored through INR, as it is a factor VII dependent metric (synthesised by the liver; circulating half-life of 6 hours). Correcting the INR with blood products therefore deprives the clinician of a prognostically important monitor of ALF trajectory and is not recommended in the absence of haemorrhage. However, vitamin $\mathrm{K}$ can be given if warfarin reversal is required or a nutritional deficiency is suspected, as these states mask the actual magnitude of liver dysfunction. Haemorrhage is rare due to the balanced reduction in pro- and anticoagulant factors. In case of haemorrhage, consultation with haematology and critical care is recommended, but platelet and fibrinogen correction alone may be adequate interventions. Sedative agents, non-steroidal anti-inflammatory drugs and intramuscular injections should also be avoided in these patients as they may precipitate coma, renal failure and haemorrhage, respectively.

\section{Primary escalation}

Early referral to critical care is recommended and must occur alongside referral to a transplant centre if hepatic encephalopathy develops. Once encephalopathy develops, deterioration can be precipitous with rapid progression to intracranial hypertension and death (Table 2). Intubation and sedation are, therefore, recommended once grade 2 or 3 encephalopathy develops, and certainly prior to any inter-hospital transfers. Hypoglycaemia and a rapidly rising INR are also red flags for ICU referral when liver injury is suspected.

\section{Definitive treatments and critical care management}

The use of NAC for POD is well established and its use in nonPOD ALF has already been discussed. Other aetiology-specific treatments that may be curative include fetal delivery for pregnancy-induced ALF, antiviral therapy for viral hepatitis and immunosuppression for autoimmune hepatitis. Considering the multidisciplinary nature of management and risk of progression, close liaison with a transplant centre is recommended for expertise as remote advice and guidance may optimise medical 
Table 2. West Haven hepatic encephalopathy criteria

\begin{tabular}{lllll} 
Grade & $\mathbf{1}$ & $\mathbf{2}$ & $\mathbf{3}$ & $\mathbf{4}$ \\
Features & $\begin{array}{l}\text { Preserved consciousness, } \\
\text { behavioural changes. }\end{array}$ & Drowsiness, disorientation, & Marked confusion. & Comatose. \\
& asterixis. & Inappropriate behaviour. & $\begin{array}{l}\text { Incoherent speech. } \\
\text { Somnolescence. }\end{array}$ & $\begin{array}{l}\text { Decerebrate or decorticate } \\
\text { posturing. }\end{array}$ \\
& & & Rouses to voice. & \\
\hline
\end{tabular}

NB There is some overlap between grades; a neat, sequential passage through grades does not always accompany progression.

management and improve the chances of successful outcome without transfer.

Detailed critical care management is outside the scope of this article, but it is important to say that critical care interventions, many of which are modified in ALF, have a significant impact on outcomes. Advanced neuroprotection in ALF involves sedation, ventilation, close control of arterial $\mathrm{CO}_{2}$ tension, defence of cerebral perfusion pressure, targeting higher plasma sodium, actively reducing ammonia levels, defending against hypoglycaemia and preventing pyrexia. Hyperacute ALF patients also develop a hyperdynamic circulation in the context of distributive shock, acute renal failure and a profound metabolic acidosis. Optimal treatment is therefore only realistically deliverable in critical care, but unusual considerations, eg around renal replacement therapy in the context of hyperammonaemia (even without acute kidney injury), also mean that local ICUs need to liaise with transplant ICUs for expertise. ${ }^{10-12}$

\section{Secondary escalation, poor prognostic criteria and transfer to a transplant centre}

Frequent feedback of patient progress to a transplant centre also minimises the risk of missing a transfer opportunity due to excessive patient instability. Patients with persistent $\mathrm{pH}<7.3$ or lactic acidosis following fluid resuscitation, climbing INR, acute kidney injury, hypoglycaemia or reduced consciousness level should be referred to a specialist centre. ${ }^{4}$ These indicate patients who are likely to meet super-urgent listing criteria. ${ }^{13}$ These criteria, drawn upon the basis of outcomes of cohorts of patients from the

Table 3. King's College hospital selection criteria for adult super-urgent liver transplantation ${ }^{14}$

Criteria for liver
transplantation in
paracetamol-induced acute
liver failure

$\mathrm{pH}<7.3$ following fluid resuscitation and $>24$ hours since paracetamol ingestion or

lactate $>3$ following

resuscitation

or

all of the following in a 24 -hour period:

INR $>6.5$ ( $\mathrm{PT}>100$ seconds)

$\mathrm{Cr}>300 \mu \mathrm{mol} / \mathrm{L}$ grade 3 or 4 encephalopathy.
Criteria for liver transplantation in non-paracetamol-induced acute liver failure or

Any three of the following variables:

$>$ age $<10$ or $>40$ years

$>$ aetiology - non-A/B hepatitis, drug induced

$>$ duration of jaundice to encephalopathy $>7$ days

$>$ INR $>3.5$ (PT $>50$ seconds)

$>$ serum bilirubin $>300 \mu \mathrm{mol} / \mathrm{L}$
INR $>6.5$ (PT >100 seconds)

$\mathrm{Cr}=$ creatinine; $\mathrm{INR}=$ international normalised ratio; $\mathrm{PT}=$ prothrombin time. 1980s, consistently deliver good specificity (82-95\%) but have poorer sensitivity (58-69\%) for mortality. ${ }^{14,15}$ There is a case for updating these criteria. ${ }^{16,17}$

Transfer to a specialist centre does not guarantee transplantation, nor survival. However, access to significant expertise that has been concentrated across only seven UK centres, access to modalities such as high-volume plasma exchange (which improves the likelihood of survival in patients who meet criteria but are unsuitable transplant candidates either through sickness or psychosocial history), and of course the potential to be listed and rapidly receive a liver transplant, undoubtedly all contribute to maximising the chance of survival once transfer criteria have been met. ${ }^{18}$

\section{Conclusions}

Acute liver failure is a rare syndrome. Early recognition and broad investigations for aetiology are required. Timely medical management as described can significantly improve survival and avoid the need for liver transplantation. Extrahepatic organ failure is common, and all patients should be discussed with the local critical care team and regional transplant centre.

\section{References}

1 Bernal W, Auzinger G, Dhawan A, Wendon J. Acute liver failure. Lancet 2010;376:190-201.

2 Ganger DR, Rule J, Rakela J et al. Acute liver failure of indeterminate etiology: a comprehensive systematic approach by an expert committee to establish causality. Am J Gastroenterol 2018;113:1319.

3 Bernal W, Lee WM, Wendon J, Larsen FS, Williams R. Acute liver failure: A curable disease by 2024? J Hepatol 2015;62(1 Suppl):S112-20.

4 European Association for the Study of the Liver. EASL clinical practical guidelines on the management of acute (fulminant) liver failure. J Hepatol 2017;66:1047-81.

5 O'Grady JG, Schalm SW, Williams R. Acute liver failure: redefining the syndromes. Lancet 1993;342:273-5 [and Corrections. Lancet 1993;342:1000].

6 Bernal W, Hyyrylainen A, Gera A et al. Lessons from look-back in acute liver failure? A single centre experience of 3300 patients. J Hepatol 2013;59:74-80.

7 Rolando N, Philpott-Howard J, Williams R. Bacterial and fungal infection in acute liver failure. Semin Liver Dis 1996;16:389-402.

8 Hu J, Zhang Q, Ren X, Sun Z, Quan Q. Efficacy and safety of acetylcysteine in "non-acetaminophen" acute liver failure: A metaanalysis of prospective clinical trials. Clin Res Hepatol Gastroenterol 2015:39:594-9.

9 Pettie JM, Caparrotta TM, Hunter RW et al. Safety and efficacy of the SNAP 12-hour acetylcysteine regimen for the treatment of paracetamol overdose. EClinicalMedicine 2019;11:11-7.

10 Murphy N, Auzinger G, Bernel W, Wendon J. The effect of hypertonic sodium chloride on intracranial pressure in patients with acute liver failure. Hepatology 2004;39:464-70.

11 Slack AJ, Auzinger G, Willars C et al. Ammonia clearance with haemofiltration in adults with liver disease. Liver Int 2014;34:42-8. 
12 Audimooolam VK, McPhail MJ, Sherwood R et al. Elevated troponin I and its prognostic significance in acute liver failure. Crit Care 2012;16:R228.

13 Neuberger ], Gimson A, Davies M et al. Selection of patients for liver transplantation and allocation of donated livers in the UK. Gut 2008:57:252-7.

14 O'Grady JG, Alexander GJ, Hayllar KM, Williams R. Early indicators of prognosis in fulminant hepatic failure. Gastroenterology 1989;97:439-45.

15 McPhail MJ, Wendon JA, Bernal W. Meta-analysis of performance of King's College Hospital Criteria in prediction of outcome in nonparacetamol-induced acute liver failure. J Hepatol 2010;53:492-9.

16 Bernal W, Wang Y, Maggs ] et al. Development and validation of a dynamic outcome prediction model for paracetamol-induced acute liver failure: a cohort study. Lancet Gastroenterol Hepatol 2016;1:217-25

17 Bernal W, Williams R. Beyond KCH selection and options in acute liver failure. Hepatol Int 2018;12:204-13.

18 Larsen FS, Schmidt LE, Bernsmeier C et al. High-volume plasma exchange in patients with acute liver failure: An open randomised controlled trial. J Hepatol 2016;64:69-78.

Address for correspondence: Dr Mohammed Asif Arshad, University Hospitals Birmingham NHS Foundation Trust, Queen Elizabeth Hospital, Mindelsohn Way, Birmingham B15 2TH, UK.

Email: mohammed.arshad@uhb.nhs.uk

\section{Mental health and wellbeing resource}

This online resource aims to shine a spotlight on the mental health and wellbeing of physicians by opening up the conversation about mental health issues and their impact.

It will help you recognise the warning signs in yourself and others, as well as know about the steps you can take to stay well and seek the right support when you need it.

www.rcplondon.ac.uk/wellbeing

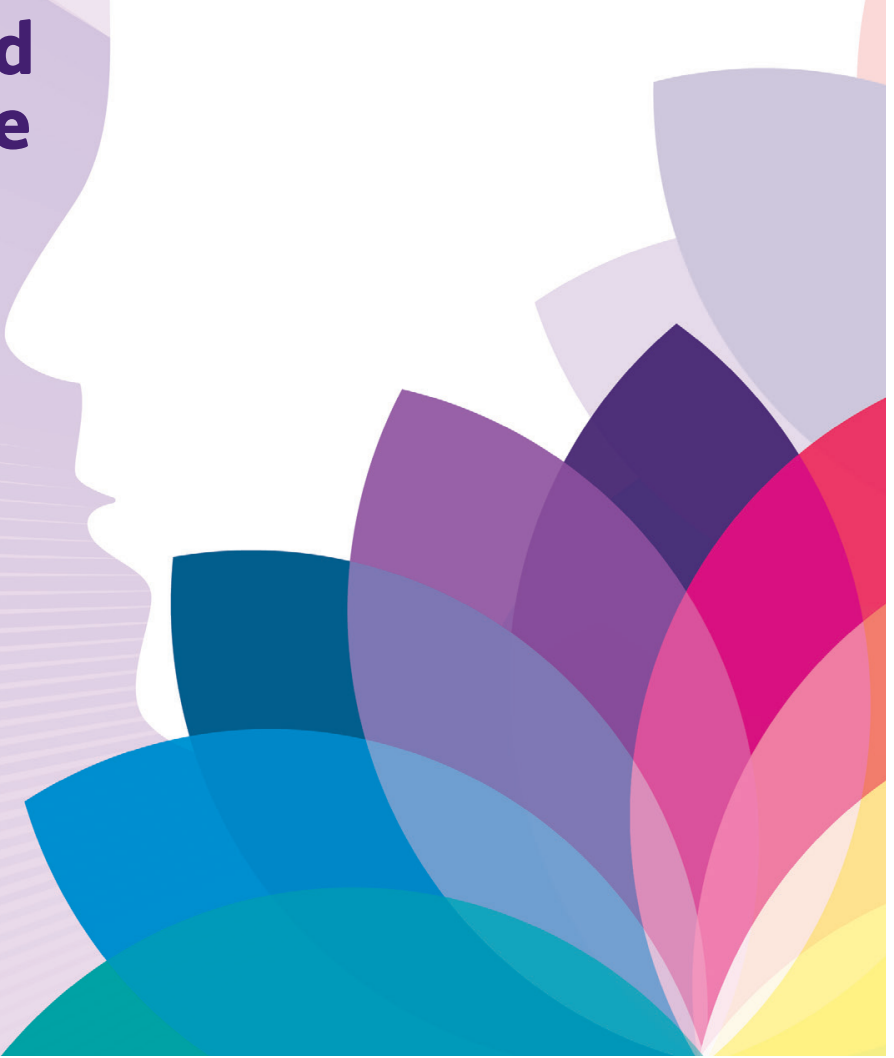

